\title{
Simulation of plastic deformation and destruc- tion in the process of chip formation
}

\author{
Tanel Tärgla ${ }^{1, *}$, Jüri Olt ${ }^{1, * *}$, and Olga Liivapuu ${ }^{1, * * *}$ \\ ${ }^{1}$ Institute of Technology, Estonian University of Life Sciences, Fr. R. Kreutzwaldi 56, 51014 Tartu, \\ Estonia
}

\begin{abstract}
Metal cutting is a complex process in which several mechanisms are at work simultaneously. The mathematical modelling allows carrying out research into the optimization of machining conditions. This work examines the simulation of chip formation during the process of cutting. The studies demonstrated that the chip formation process, taking into account the plastic deformation and destruction of metal in the local zone, is most appropriately represented by a rheological model in the form of a series connection of elasticductile-plastic relaxing medium of Ishlinskiy (reflecting the process of primary deformation of metal from the cut off layer) and the medium of Voigt with two elastic-dissipative elements (representing the process of deformation and frictions from the convergent shaving). The attained complex rheological model served as the basis for constructing a representative dynamic model for the chip formation process. The key factors that govern the chip formation have been taken into account, such as tool vibration frequency and amplitude, depth of cut, feed rate.
\end{abstract}

\section{Introduction}

The process of chip formation proceeds in a relatively small area with a high strain rate, with a very low relative deformation of the material $[1,2]$. Physical properties of deformed metal in the process of cutting can be described with the help of discrete rheological models represented by a certain set of mechanical elements: elasticity, viscosity and plasticity. Many real metals combine behaviours of all these elements and it is possible to consider the stressstrain state of metal under the action of external loads. Maxwell and Voigt models [3] are adequate for qualitative analyses, but generally poor for the representation of behaviour of real materials. In order to describe the nature of changes in stress and strain of metal and their dependence on metal structure, we need to increase the number of parameters in model [4]. In this paper in accordance with the results of previous research [5-7] we describe the formation of flat chip in the process of cutting as interaction between the cutting wedge and the workable material. Our model is constructed in two stage up to the moment of the formation of chip, i.e. the deformation to the shear plane and the shift of the thin stripe of metal on the plane of shift.

\footnotetext{
*e-mail: tanel.targla@emu.ee

**e-mail: jyri.olt@emu.ee

***e-mail: olga.liivapuu@emu.ee
} 


\section{Analysis of the stability of the chip formation process}

We describe the process of chip formation with the help of the rheological model proposed by Veitz and Maksarov [8]. This model is constructed of the elastic-ductile-plastic medium of Ishlinskiy type $[9,10]$ and viscoelastic Voigt medium with the delay of deformation connected in series (Fig. 1). The force $\mathbf{P}$ applied to the point $D$ imitates by the linear elastic spring element $H_{1}$ the elastic instantaneous deformation, formed in front of the area of plastic deformation in the zone of chip formation. The next part of Ishlinskiy medium consists of the spring $\mathrm{H}_{2}$ and the dash-pot $\mathrm{N}_{2}$ connected in parallel. It imitates the prolonged viscoelastic deformation. After reaching the yield stress $\sigma_{y}$, which imitates St. Venant element $S t . V$, the instantaneous residual deformation appears. This leads to the shaping of local segments of shift in the primary deformation zone of the cut off layer. At this stage, permanent, irreversible deformations begins. The shift of material moves foreword in the direction of tool movement and this prolonged viscoelastic deformation can be described by Voigt model consisting of the spring $\mathrm{H}_{3}$ and the dash-pot $\mathrm{N}_{3}$ in parallel.

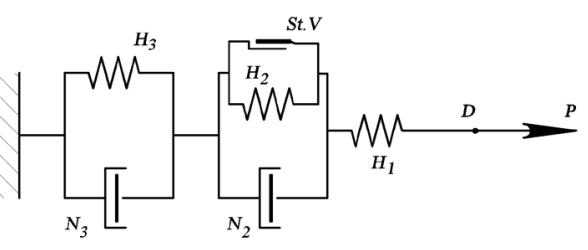

Figure 1. The rheological model of chip formation in the process of cutting [10]

Fig. 1 represents the model, which connects the process of brittle and ductile fracture of material. Indeed, we can assume that the brittle fracture corresponds to break of elastic external element $H_{1}$, and viscous fracture corresponds to the break of elastic internal element $H_{2}$, which is preceded by the relative shift of viscous element $N_{2}$ [10]. Elements of mechanical system can be considered in relation to the direction of deformation with the input and output, the direction of the pointer in the figure 1 indicates the direction of deformation of the system.

The differential constitutive relations for the model describing the process of chip formation can be presented in the form of the following rheological equation [11]:

$$
a_{1} \ddot{\sigma}+a_{2} \dot{\sigma}+a_{3}\left(\sigma \pm \sigma_{y}\right)=a_{4} \ddot{\varepsilon}+a_{5} \dot{\varepsilon}+a_{6} \varepsilon
$$

where coefficients $a_{i},(i=1,2, \ldots, 6)$ depend on stiffness coefficients and coefficients of linear resistance, $\sigma_{y}$ is a yield stress.

Let us show the relations between coefficients of the rheological equation (1) and the stiffness and viscosity of material of the mechanical system. To this end we represent the developed model using integral formulation and the Laplace transform. Let $p^{i}=\frac{\partial^{i}}{\partial t^{i}}$. It is possible to determine the operator resistance of the Ishlinskiy model in the form

$$
z_{I}(p)=\frac{p}{c_{1}}+\frac{p}{\beta_{2} p+c_{2}}
$$

where the properties of elastic elements $H_{1}$ and $H_{2}$ of the Ishlinskiy model are described by stiffness coefficients $c_{1}$ and $c_{2}$ and the dash-pot element $N_{1}$ is characterized by the coefficient of linear resistance $\beta_{2}$. 
For the model of Voigt the operator resistance takes the form

$$
z_{V}(p)=\frac{p}{\beta_{3} p+c_{3}}
$$

where $H_{3}$ and $N_{3}$ are described by the stiffness coefficient $c_{3}$ and the coefficient of linear resistance $\beta_{3}$ respectively.

For the entire model describing the process of chip formation we get

$$
\begin{aligned}
z(p) & =\frac{\beta_{2} p^{2}+c_{2} p+c_{1} p}{\beta_{2} c_{1} p+c_{1} c_{2}}+\frac{p}{\beta_{3} p+c_{3}} \\
& =p \cdot \frac{\beta_{2} \beta_{3} p^{2}+\left(\beta_{2} c_{1}+\beta_{2} c_{3}+\beta_{3} c_{1}+\beta_{3} c_{2}\right) p+\left(c_{1} c_{2}+c_{2} c_{3}+c_{1} c_{3}\right)}{\beta_{2} \beta_{3} c_{1} p^{2}+\left(\beta_{2} c_{1} c_{3}+\beta_{3} c_{1} c_{2}\right) p+c_{1} c_{2} c_{3}}
\end{aligned}
$$

In order to simplify the calculations, we introduce the following notations for the coefficients of differential operator $p$ and free terms in the numerator and denominator of equation (2):

$$
\begin{aligned}
& a_{1}=\beta_{2} \beta_{3} \\
& a_{2}=\beta_{2} c_{1}+\beta_{2} c_{3}+\beta_{3} c_{1}+\beta_{3} c_{2} \\
& a_{3}=c_{1} c_{2}+c_{2} c_{3}+c_{1} c_{3} \\
& a_{4}=\beta_{2} \beta_{3} c_{1} \\
& a_{5}=\beta_{2} c_{1} c_{3}+\beta_{3} c_{1} c_{2} \\
& a_{6}=c_{1} c_{2} c_{3}
\end{aligned}
$$

Then the operator resistance can be expressed in the form [10]

$$
z(p)=p \cdot \frac{a_{1} p^{2}+a_{2} p+a_{3}}{a_{4} p^{2}+a_{5} p+a_{6}}
$$

it means that returning to the space of originals we obtain the rheological equation (1), which define the relation between stress and deformation of the mechanical system.

To motivate the adequacy of the rheological model (1) we assume that the deformation in time is supposed to be known and solve this equation for the stress. We substitute $\sigma$ in the equation (1) by a new variable $x=a_{3}\left(\sigma \pm \sigma_{y}\right)$ and get

$$
\ddot{x}+2 \gamma \dot{x}+\omega_{0}^{2} x=f(t)
$$

where

- $\omega_{0}=\sqrt{\frac{a_{3}}{a_{1}}}=\sqrt{\frac{c_{1} c_{2}+c_{2} c_{3}+c_{1} c_{3}}{\beta_{2} \beta_{3}}}$ is a frequency,

- $\gamma=\frac{a_{2}}{2 a_{1}}=\frac{\beta_{2} c_{1}+\beta_{2} c_{3}+\beta_{3} c_{1}+\beta_{3} c_{2}}{2 \beta_{2} \beta_{3}}$ is a damping constant,

- $f(t)=f(\varepsilon, \dot{\varepsilon}, \ddot{\varepsilon})=\frac{a_{3} a_{4} \ddot{\varepsilon}+a_{3} a_{5} \dot{\varepsilon}+a_{3} a_{6} \varepsilon}{a_{1}}$.

Let us denote the vibration frequency by $\omega=\sqrt{\gamma^{2}-\omega_{0}^{2}}$. The solutions of homogeneous part of equation (3) are

- for oscillation mode $x(t)=e^{-\gamma t}(A \cos \omega t+B \sin \omega t)$, when $\gamma^{2}-\omega_{0}^{2}<0$,

- for relaxation mode $x(t)=A e^{(-\gamma+\omega) t}+B e^{(-\gamma-\omega) t}$, when $\gamma^{2}-\omega_{0}^{2}>0$.

The general solution of equation (3) for relaxation mode is

$$
x(t)=e^{-\gamma t}\left[\frac{1}{2} e^{\omega t} \cdot \int \frac{f(t) e^{(\gamma-\omega) t}}{\omega} d t-\frac{1}{2} e^{-\omega t} \cdot \int \frac{f(t) e^{(\gamma+\omega) t}}{\omega} d t+A e^{\omega t}+B e^{-\omega t}\right]
$$


With a fixed value of deformation $\varepsilon=\varepsilon_{0}$ function $f(t)$ can be expressed as follows

$$
f(t)=\frac{a_{3} a_{6} \varepsilon_{0}}{a_{1}} .
$$

After transition from variable $x$ to variable $\sigma$ and taking into account initial conditions $\sigma(0)=$ $\sigma_{0}$, and $\dot{\sigma}(0)=$ const , an equation describing stress relaxation takes the following form

$$
\begin{aligned}
\sigma(t) & =\sigma_{y}+\frac{a_{6} \varepsilon_{0}}{2 \omega a_{1}} \cdot\left(\frac{1}{\gamma-\omega}-\frac{1}{\gamma+\omega}\right)+ \\
& +\frac{1}{2 \omega}\left[\left(\sigma_{0}-\sigma_{y}\right)\left((\gamma+\omega) e^{\omega t}+(\omega-\gamma) e^{-\omega t}\right)-\frac{a_{6} \varepsilon_{0}}{a_{1}} \cdot\left(\frac{1}{\gamma-\omega} e^{\omega t}-\frac{1}{\gamma+\omega} e^{-\omega t}\right)+\right. \\
& \left.+\dot{\sigma}(0)\left(e^{\omega t}-e^{-\omega t}\right)\right] \cdot e^{-\gamma t} .
\end{aligned}
$$

From equation (5) we can conclude that for our rheological model stress relaxes to its equilibrium with two relaxation times $\tau_{1}=\frac{1}{\gamma-\omega}$ and $\tau_{2}=\frac{1}{\gamma+\omega}$. We get the following expression for the for the rates of relaxation $\gamma-\omega$ and $\gamma+\omega$ in the terms of stiffness coefficients $c_{i}$ and coefficients of linear resistance $\beta_{i}$ :

$$
\begin{aligned}
\gamma \pm \omega & =\frac{a_{2}}{2 a_{1}} \pm \sqrt{\frac{a_{2}^{2}-4 a_{1} a_{3}}{4 a_{1}^{2}}}=\frac{1}{2 a_{1}}\left(a_{2} \pm \sqrt{a_{2}^{2}-4 a_{1} a_{3}}\right)= \\
& =\frac{1}{2 \beta_{2} \beta_{3}}\left(\left(\beta_{2} c_{1}+\beta_{2} c_{3}+\beta_{3} c_{1}+\beta_{3} c_{2}\right) \pm\right. \\
& \pm \sqrt{\left.\left(\beta_{2} c_{1}+\beta_{2} c_{3}+\beta_{3} c_{1}+\beta_{3} c_{2}\right)^{2}-4 \beta_{2} \beta_{3}\left(c_{1} c_{2}+c_{2} c_{3}+c_{1} c_{3}\right)\right)} .
\end{aligned}
$$

\section{Conclusions}

It follows from proposed rheological model in the zone of primary plastic deformation occur processes which generate instability of chip formation. The analysis of the developed plastic deformation of metal shows that this process has wavelike nature. At the moment which corresponds to the implementation of plastic deformation in the zone of plastic contact of chip with the tool, which reflects the elastic reaction of the workable material, the cut off layer undergoes the secondary deformation. Our theoretical studies were confirmed by experimental data which were conducted in the broader band for back rake angle $\gamma$ from $-5^{\circ}$ to $25^{\circ}$ and for different values of cutting speed. It was determined in [9] that the form of chip by processing steel $41 \mathrm{Cr} 4$ is following:

- discontinuous chip for $\gamma=-5^{\circ}$ and cutting speed $10 \mathrm{~m} / \mathrm{min}$;

- built-up edge chip for $\gamma=25^{\circ}$ and cutting speed $25 \mathrm{~m} / \mathrm{min}$;

- continuous chip for for $\gamma=10^{\circ}$ and cutting speed $75 \mathrm{~m} / \mathrm{min}$.

It confirms our studies on the origin of the primary relaxation phenomena, which induce the origination of the self-oscillating process by turning materials.

\section{References}

[1] P. Albertelli, N. Cau, G. Bianchi, M. Monno, Int. J. Adv. Manuf. Tech.58, 10, (2012)

[2] F. Klocke, W. Lortz, D. Trauth, Int. J. Mech. Sci. 135, 12 (2018)

[3] R. Christensen, Theory of Viscoelasticity (Dover Publications Inc, New York, 2010) 
[4] A. Bobryshev, A. Lakhno, P. Voronov, E. Galimov, R. Sharafutdinov, N. Galimova, IOP Conf. Ser.: Mater. Sci. Eng. 86, 7 (2015)

[5] V. Maksarov, M. Madissoo, J. Olt, J. Mech. and. Ind. Res. 1, 7 (2013)

[6] J. Olt, O. Liivapuu, V. Maksarov, A. Liyvapuu, T. Tärgla, Engineering Mathematics I (Springer Proceedings in Mathematics and Statistics, New York, 2016)

[7] V. Maksarov, A. Khalimonenko, J. Olt,JSBCM 69, 6 (2017)

[8] V. Veitz, V. Maksarov, Dynamics and control of the process of chip formation with cutting-edge mechanical processing (NWPI, Sant-Petersburg, 2000) $160 \mathrm{p}$.

[9] V. Maksarov, J. Olt, T. Laatsit, T. Leemet, Proceedings of 6th International DAAAM Baltic Conference INDUSTRIAL ENGINEERING (Tallinn, 2008) pp. 263-268

[10] J. Olt, A. Liyvapuu, M. Madissoo, V. Maksarov, IJMPT 53, 14 (2016)

[11] J. Olt, V. Maksarov, Proceedings of the DAAAM symposium (Vienna, 2015) pp. 229-237 